\title{
Severity of alcoholic liver disease and markers of thyroid and steroid status
}

\author{
P. Burra' ${ }^{1}$ J.A. Franklyn, D.B. Ramsden, E. Elias ${ }^{1}$ and M.C. Sheppard
}

Department of Medicine, University of Birmingham and 'Liver Unit, Queen Elizabeth Hospital, Edgbaston, $U K$

Summary: Alcoholic liver disease is associated with abnormalities in circulating levels of thyroid, adrenal and gonadal steroid hormones. The relative importance of ethanol consumption and severity of liver disease in the aetiology of these changes and their relationship to clinical abnormalities are unclear. We studied 31 subjects with alcohol-induced liver disease divided into three groups according to the severity of histological features: fatty change, hepatitis and cirrhosis.

Circulating concentrations of thyroid, adrenal and gonadal steroid hormones, together with their major binding proteins, were measured in all subjects, and changes related to histology and tests of liver function, as well as clinical endocrine status. A reduction in circulating free tri-iodothyronine (fT3) was seen in subjects with alcoholic hepatitis and cirrhosis, in association with normal or reduced levels of thyrotrophin (TSH).

The absence of abnormalities in subjects with fatty change despite similar ethanol intake to the other groups, and correlations between $\mathrm{fT} 3$ and liver function tests, suggest that changes in $\mathrm{fT} 3$ reflect the severity of underlying liver disease. Similarly, marked increases in circulating cortisol in the hepatitis and cirrhosis groups, and correlations between cortisol and liver function, suggest that changes largely reflect hepatic disease. The absence of clinical features of hypothyroidism or Cushing's syndrome in these groups, despite abnormalities of $\mathrm{fT} 3$ and cortisol, suggest an altered tissue sensitivity to hormone effects. Int contrast, increases in circulating oestradiol and reductions in testosterone were found in all three groups info males. These findings suggest that both direct effects of ethanol and hepatic dysfunction determine changes in gonadal steroids in males.

\section{Introduction}

The liver, a primary site of ethanol-related damage, plays an important role in thyroid hormone metabolism, being involved in the conjugation and deiodination of thyroxine (T4) and tri-iodothyronine (T3), as well as the synthesis and secretion of the major thyroid hormone-binding proteins, thyroxine-binding globulin (TBG), thyroxine-binding prealbumin, and albumin. ${ }^{1,2}$ Furthermore, the liver is an important target organ for thyroid hormone action, with a larger number of gene products expressed in the liver being responsive to $\mathrm{T} 3 .^{3}$

In liver disease in general, serum total and free T3 concentrations are often reduced, changes associated with an elevation in serum reverse T3 (3,3', $5^{\prime}$-tri-iodothyronine) (rT3). Serum total and free $\mathrm{T} 4$ concentrations are often $\mathrm{low}^{4}$ but may be elevated or unchanged. ${ }^{5,6}$ Despite these changes in

Correspondence: Professor M.C. Sheppard, Ph.D., F.R.C.P., Department of Medicine, Queen Elizabeth Hospital, Edgbaston, Birmingham B15 2TH, UK.

Accepted: 16 March 1992 circulating thyroid hormones, serum thyrotrophin (TSH) concentrations remain within the normal range in the majority of patients with chronic liver disease. $^{7-9}$

In addition to changes in circulating concentrations of thyroid hormones in liver disease, ${ }^{10}$. alcoholic liver disease is commonly associated with $\frac{\text { 의 }}{3}$ abnormalities of gonadal steroids, even before the

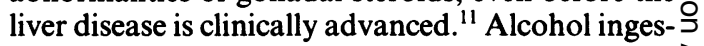
tion alone can result in severe gonadal failure in $\frac{7}{0}$ both men and women in the absence of any? alcoholic liver disease. ${ }^{12}$ The described effects of ${ }_{\sigma}$ ethanol, and its metabolic product acetaldehyde, include enhanced testosterone degradation and $\mathcal{N}^{\circ}$ conversion to oestrogens, as well as decreased $\omega$ testicular steroid synthesis. ${ }^{13,14}$ These changes in circulating gonadal steroids may be associated with clinically evident hypogonadism in both males and females. ${ }^{15,16}$

In addition, signs of adrenocortical excess have $\frac{0}{0}$ been described in patients with alcoholic liver $\frac{\vec{D}}{\mathrm{D}}$ disease. ${ }^{17-19}$ Despite the well-known association of $\frac{\mathbb{Q}}{\widetilde{Q}}$ liver disease with endocrine abnormalities, pre- $\stackrel{\mathbb{Q}}{0}$ 
vious studies of endocrine status in alcoholic liver disease have examined heterogeneous groups of patients who have often been classified poorly in terms of pathological diagnosis. In the present study, we have examined in detail three groups of patients with alcohol-induced liver disease, with carefully defined histological features, and have compared and contrasted the endocrine state of such patients in order to relate abnormalities to the severity of disease and to elucidate the likely aetiological roles of alcoholic ingestion and secondary hepatic dysfunction.

\section{Subjects and methods}

Thirty-one patients, 21 males and 10 females, aged 25-74 years, with alcoholic liver disease, were studied. The mean duration of drinking was 8.3 years (range 2-20), the mean intake was $237 \mathrm{~g}$ alcohol/day (range 20-900). Twenty patients were drinking at the time of hospital admission, 11 had abstained for between 1 and 7 days prior to hospital admission. The diagnosis of liver disease was made in all cases according to the criteria selected by an international liver research group published in 1981 and widely applied. ${ }^{20}$ Nine patients were defined as having fatty change in the liver, ten patients acute hepatitis and 12 patients cirrhosis (two Child B and ten Child C). None of the patients with fatty change in the liver had ascites, spider naevi, hepatic encephalopathy or previous history of gastrointestinal bleeding. Six patients with alcoholic hepatitis had jaundice and ascites, four spider naevi and two encephalopathy. Ten patients with cirrhosis had jaundice and ascites, eight of whom also had spider naevi and three encephalopathy. Three patients with alcoholic hepatitis bled from the gastrointestinal tract during the end-stage of their liver disease due to poor coagulation and two patients with cirrhosis had a previous history of bleeding from oesophageal varices.

Clinical features of thyroid disease, such as goitre, eye signs, tremor, sweating and abnormal relaxation of reflexes were sought in all patients. Clinical symptoms and signs of Cushing's syndrome such as proximal myopathy, truncal obesity, facial erythema and hypertension were also sought. Two female patients were postmenopausal, none had undergone hysterectomy.

All the patients have been assessed and evaluated for signs of liver, thyroid, adrenal or gonadal dysfunction by the same investigator.

Liver and renal function tests were performed in all subjects as markers of severity of liver disease (aspartate aminotransferase (AST), gamma-glutamyl transferase (GGT), alkaline phosphatase (Alk$\mathrm{Ph}$ ), prothrombin time/control prothrombin time (INR), albumin (ALB), bilirubin (BIL), urea and creatinine (CREAT)). Serum samples for hormone assays were stored at $-20^{\circ} \mathrm{C}$. Serum-free T4 (fT4) (normal range 9.8-22 pmol/1), free T3 (fT3) (normal range 4.3-9.4 pmol/l), thyrotrophin (TSH) (normal range 0.4-6.9 mU/1), total T4 (tT4) (normal range $65-160 \mathrm{nmol} / \mathrm{l}$ ) were measured using Amerlite chemiluminescent immunoassays (Amerlite, Amersham International Plc). Serum cortisol (normal range $200-700 \mathrm{nmol} / \mathrm{l}$ ), oestradiol (normal range: male $90-220 \mathrm{pmol} / \mathrm{l}$; female $160-2220$ pmol/1), testosterone (normal range: male 11-36 $\mathrm{nmol} / \mathrm{l}$; female $0.8-3.1 \mathrm{nmol} / \mathrm{l}$ ) levels were determined by in-house radioimmunoassay. Thyroxine binding globulin levels (TBG) (normal range $5-20 \mathrm{mg} / \mathrm{l}$ ) were measured using a radial immunodiffusion kit (University of Birmingham), sex hormone binding globulin (SHBG) levels were measured using a Pharmacia immunoradiometric assay (normal range: male 11-71; female 20-118) and cortisol binding globulin (CBG) levels (normal range $20-50 \mathrm{mg} / \mathrm{l}$ ) were measured using a radial immunodiffusion kit (University of Birmingham).

The statistical analysis of data was carried out using non-parametric analysis and correlations were obtained using Rank Correlation analysis (Kendall tau).

\section{Results}

The liver and renal function tests of patients studied, divided according to histological diagnosis, are shown in Table I. In the fatty liver group, the median values for all liver and renal function tests, with the exception of AST and GGT, were in the normal range while in hepatitis and cirrhosis all median values were abnormal with the exception of alkaline phosphatase which was at the upper limit. The median alcohol consumption expressed in grams of alcohol per day was significantly greater in the cirrhotic group (320; range 80-560) compared with fatty liver (100; range 20-400) and with alcoholic hepatitis (160; range 50-900).

\section{Clinical and biochemical assessment of thyroid status}

Two patients exhibited clinical features of thyroid disease, one of whom with alcoholic hepatitis had exophthalmos and another female with alcoholic cirrhosis who was clinically hypothyroid. All the remaining patients were clinically euthyroid. Thyroid function tests divided by histological groups are summarized in Table II. The median values for tests of thyroid function were within the normal range in the fatty liver group while alcoholic hepatitis patients had low fT3, and cirrhosis subjects high fT4, although an elevation in TT4/TBG 
Table I Liver and renal function tests divided by histological groups (median and range): fatty change (FC), alcoholic hepatitis (AH) and cirrhosis (AC). Data were compared using non-parametric analysis

\begin{tabular}{lcccc}
\hline & $F C$ & $A H$ & $A C$ & $\begin{array}{c}\text { Normal } \\
\text { ranges }\end{array}$ \\
\hline AST* & $54(27-170)$ & $129(25-296)$ & $98(26-252)$ & $5-30 \mathrm{u} / 1$ \\
GGT & $289(48-1830)$ & $139(85-730)$ & $172(28-1125)$ & $7-35 \mathrm{u} / 1$ \\
AlkPh & $249(148-445)$ & $341 \dagger(187-670)$ & $316(170-812)$ & $70-350 \mathrm{u} / 1$ \\
INR & $0.9(0.8-1.2)$ & $1.2 \dagger(0.8-2.3)$ & $1.4(1.0-3.2)$ & Up to 1.2 \\
ALB & $41(25-48)$ & $32 \pm(27-44)$ & $28(17-39)$ & $34-51 \mathrm{~g} / 1$ \\
BIL & $14(6-60)$ & $253(7-769)$ & $114(8-776)$ & $1-26 \mu \mathrm{mol} / 1$ \\
Urea & $4.4(2.9-8.2)$ & $8.8(1.3-50.3)$ & $10(2.4-53.4)$ & $3.4-7.6 \mathrm{mmol} / 1$ \\
CREAT & $90(70-111)$ & $145(64-707)$ & $126(47-1044)$ & $50-125 \mu \mathrm{mol} / 1$ \\
\hline
\end{tabular}

*For these abbreviations, see text; †vs FC, $P<0.05$; $\ddagger$ s $\mathrm{AC}, P<0.05$.

Table II Thyroid function tests divided by histological groups (median and range): fatty change (FC), alcoholic hepatitis (AH) and cirrhosis (AC). Data were compared using non-parametric analysis

\begin{tabular}{|c|c|c|c|}
\hline & $F C$ & $A H$ & $A C$ \\
\hline $\begin{array}{c}\text { Free T4 } \\
(\mathrm{pmol} / \mathrm{l})\end{array}$ & $17.1(13-43.4)$ & $16.9 *(7.1-95)$ & $24.3(11.8-35.5)$ \\
\hline $\begin{array}{c}\text { Free T3 } \\
(\mathrm{pmol} / \mathrm{l})\end{array}$ & $6.03(3.47-6.59)$ & $2.5+(1.43-8.84)$ & $3.05(0.5-7.44)$ \\
\hline $\begin{array}{l}\text { TSH } \\
(\mathrm{mU} / \mathrm{l})\end{array}$ & $1.5(0.7-2.26)$ & $1.73(0.1-6.74)$ & $1.8(0.09-3.3)$ \\
\hline $\begin{array}{l}\mathrm{tT} 4 \\
(\mathrm{nmol} / \mathrm{l})\end{array}$ & $100.8(58.7-284.7)$ & $52.25 \dagger(16.4-185.1)$ & $74.15(23-218.7)$ \\
\hline $\begin{array}{l}\text { TBG } \\
(\mathrm{mg} / \mathrm{l})\end{array}$ & $9.2(6-13.7)$ & $6.35 \ddagger(3.8-12.5)$ & $9.55(4.5-14.3)$ \\
\hline tT4/TBG & $10.8(6.3-27.3)$ & $8.9 \ddagger(3.5-19.8)$ & $8.9(5.3-23.3)$ \\
\hline
\end{tabular}

${ }^{*} v s$ AC, $P<0.05 ; \uparrow v s$ FC, $P<0.005 ; \ddagger v s$ FC, $P<0.05$.

ratio was not found. Two of nine patients $(22 \%)$ with fatty liver, one of ten with acute hepatitis and seven of $12(58 \%)$ with cirrhosis had fT4 levels above the normal range; one patient with hepatitis had a fT 4 concentration below the normal range. In contrast, no patient had a fT3 value above normal. Two of nine patients $(22 \%)$ with fatty liver, six of ten $(60 \%)$ with hepatitis and eight of 12 $(66 \%)$ with cirrhosis had fT3 levels below the normal range. TSH levels in the majority of patients were normal. Only two patients with hepatitis $(20 \%)$ and two with cirrhosis $(16 \%)$ had TSH levels below normal; no patient had a high TSH. Correlations between tests of thyroid function and biochemical markers of hepatic dysfunction were sought. No correlation between circulating fT4 and TSH levels with any of the liver function tests examined (albumin, bilirubin, prothrombin time ratio) were found. In contrast, there was a positive correlation between fT3 levels and albumin (tau $=0.42 ; P<0.001$ ) (Figure 1), and negative correlations between fT 3 and bilirubin (tau $=0.44 ; P<0.0005)$ and prothrombin time ratio (INR) (tau $=0.38 ; P<0.005)$. Correlations between cortisol levels and fT4, fT3 and TSH were also sought. No correlation between cortisol levels and circulating fT4 was found. In contrast there were negative correlations between cortisol levels and fT3 (Figure 1) (tau $=-0.32 ; P<0.01)$ and TSH $($ tau $=-0.25 ; P<0.03)$.

\section{Clinical and biochemical assessment of adrenal steroid status}

Only one patient of the 31 studied (the same female who had exophthalmos) had any features of Cushing's syndrome; she was noted to have a Cushingoid face and proximal myopathy. Adrenal and gonadal steroid levels divided according to histological diagnosis are shown in Table III. Median cortisol levels were normal in the fatty liver group in contrast to the median value in subjects with hepatitis which was above the normal range; 
similarly the median value in the cirrhotic group was higher than in the fatty liver group. This effect on serum cortisol did not simply reflect changes in
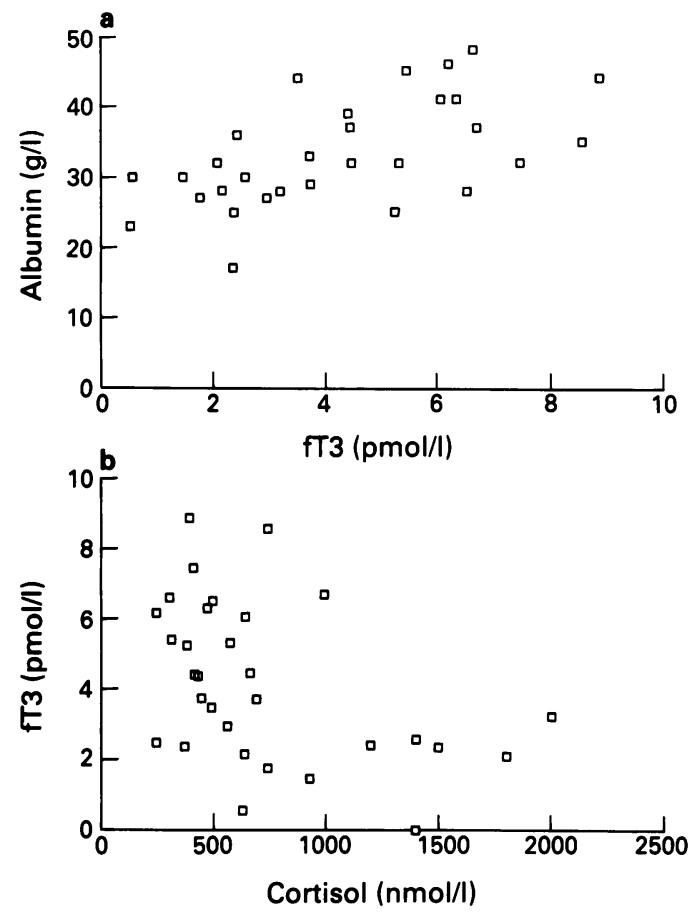

Figure 1 Correlations between free T3 and albumin (a) $(P<0.001)$ and between free T3 and cortisol (b) $(P<0.01)$. Correlations were determined using rank correlation analysis (Kendall tau). circulating CBG since the cortisol/CBG ratio demonstrated a similar pattern. All but one of the patients with fatty liver had a random cortisol level within the normal range, while six patients of ten $(60 \%)$ with hepatitis and four of $12(33 \%)$ with cirrhosis had random cortisol values above the normal range. There was a negative correlation between circulating cortisol and albumin (tau $=$ $-0.29 ; P<0.05$ ), and positive correlations between cortisol and bilirubin ( $\operatorname{tau}=0.33, P<0.01)$ and with INR (tau $=0.23 ; P=0.05)$.

\section{Clinical and biochemical assessment of gonadal steroid status}

Marked hypogonadism was evident in all of the male patients with chronic alcoholic cirrhosis; these features were not seen in subjects with fatty liver and hepatitis. The serum oestradiol and testosterone levels are shown in Table III. Median oestradiol levels were above the normal value in males in all three histological groups, with similar findings for the oestradiol/SHBG ratio. All females had normal oestradiol values. Median testosterone concentrations were markedly below the normal range in males with hepatitis and cirrhosis and normal in males with fatty liver and in all female groups; however, the median testosterone/SHBG ratio was similar in those with fatty change and hepatitis, although this ratio was low in patients with cirrhosis. In the female group, four of eight patients with hepatitis $(50 \%)$ had oestradiol levels below the normal range. Six of eight males $(75 \%)$ with fatty liver, one of two with hepatitis and all the patients with cirrhosis had circulating oestradiol

Table III Cortisol, oestradiol and testosterone levels divided by histological groups (median and range): fatty change (FC), alcoholic hepatitis (AH) and cirrhosis (AC). Data were compared using non-parametric analysis

\begin{tabular}{lccc}
\hline & $F C$ & $A H$ & $A C$ \\
\hline $\begin{array}{l}\text { Cortisol } \\
(\text { nmol/l) }\end{array}$ & $470(240-690)$ & $740^{*}(240-1800)$ & $635(370-2000)$ \\
$\begin{array}{l}\text { Cortisol/CBG } \\
\text { Oestradiol }\end{array}$ & $14.2(11.6-18.5)$ & $27.05 \ddagger(6.1-79.5)$ & $27.4^{*}(14-102.5)$ \\
$\quad \begin{array}{l}\text { Males } \\
\text { Females }\end{array}$ & $245(130-400)$ & $280(210-350)$ & $290 \dagger(240-720)$ \\
$\begin{array}{l}\text { Oestradiol/SHBG } \\
\text { Males }\end{array}$ & 1100 & $175(100-1600)$ & 1200 \\
$\quad$ Females & $6(2.9-22)$ & $15.2(14.4-16.1)$ & $10.7(2.2-71.2)$ \\
$\begin{array}{c}\text { Testosterone } \\
\text { (nmol/l) }\end{array}$ & 9.2 & $13.5(1.2-82.2)$ & 51.7 \\
$\quad$ Males & & & $3.1 \dagger(0.9-27)$ \\
Females & $15.5(11-43)$ & $8.3(0.6-16)$ & 3.1 \\
Testosterone/SHBG & 2.5 & $1.25(0.6-4.5)$ & $0.15 \dagger) 0.04-0.5)$ \\
Males & $0.47(0.13-1.56)$ & $0.35(0.04-0.66)$ & 0.13 \\
Females & 0.02 & $0.06(0.02-7.45)$ & \\
\hline
\end{tabular}

${ }^{*} v s$ FC, $P<0.05$; †vs FC, $P<0.01$; $v s$ FC, $P<0.06$ 
above normal. Three of eight females $(37.5 \%)$ with hepatitis had testosterone below the normal range, only one was above normal. Nine of 21 males were found to have testosterone concentrations lower than normal, including one of two with hepatitis and eight of $11(72.7 \%)$ with cirrhosis. One patient with fatty liver had a serum testosterone value above normal. Correlations between levels of both circulating oestradiol and testosterone with liver function tests were sought. Concentrations of oestradiol and testosterone in males demonstrated a negative correlation with serum albumin (tau $=$ $-0.32 ; P<0.05-$ tau $=0.38 ; P<0.05$ ). In contrast in females, no correlation between gonadal steroids and tests of liver function was seen.

\section{Discussion}

We have examined clinical and biochemical changes in endocrine status in a large group of alcoholic liver disease patients with histologically defined liver pathology and have related endocrine changes to the severity of liver disease in order to examine the underlying mechanisms accounting for endocrine dysfunction.

In the majority of patients examined marked changes were found in circulating thyroid hormones in the absence of significant changes in TSH, in accord with other reports. ${ }^{10,21}$ Despite clinical features of euthyroidism in all but one and although TSH was normal or low in all the subjects examined, in almost half of our patients with alcoholic liver disease, free T3 values fell below the normal range. This abnormality of circulating T3 has been reported in patients with a wide variety of 'non-thyroidal' illnesses such as myocardial infarction, starvation and renal failure, as well as in association with liver disease. ${ }^{8}$ It has been postulated that the decrease in circulating T3 found in liver disease, as in other illnesses, reflects decreased formation of $\mathrm{T} 3$ from $\mathrm{T} 4$ secondary to a decrease in the monodeiodination of the outer ring of $\mathrm{T} 4 \mathrm{by}$ the enzyme 5 '-monodeiodinase. ${ }^{22}$ We demonstrated a negative correlation between fT3 and cortisol levels, a finding in accord with the view that glucocorticoids may inhibit peripheral conversion of T4 to T3 ${ }^{23-27}$ Reductions in circulating T3 were seen most frequently in patients with alcoholic hepatitis or cirrhosis, but were uncommon in those with fatty liver with only mild liver dysfunction, despite a similar level of ethanol consumption in the fatty liver and hepatitis groups. In support of the view that the decrease in $\mathrm{T} 3$ is related to the severity of the liver disease, significant correlations between fT3 levels and liver function tests (albumin, bilirubin, prothrombin time ratio) were seen. Israel $e t$ al. have reported previously in a group of alcoholic patients ${ }^{10}$ a significant inverse correlation between T3 levels and the severity of liver disease, determined by histology and liver function tests, as well as a progressive increase in $\mathrm{T} 3$ in those patients displaying a favourable outcome, suggesting that $\mathrm{T} 3$ concentrations may be considered as a prognostic factor. Our findings confirmed that T3 levels may be considered a sensitive index of hepatic function in alcoholic liver disease.

Of interest in the current series is the number of patients with $\mathrm{fT} 4$ above normal, changes seen in the absence of suppressed TSH. In contrast to the decrease in $\mathrm{fT} 3$ seen in the majority of the patients, fT4 was found to be above normal in a third of the patients studied. In relation to the histology, this abnormality was seen in $22 \%$ of patients with fatty liver but over half of patients with cirrhosis had fT4 concentrations above the normal range. This in creased fT4 may reflect impaired deiodination of T4 to T3 or impaired degradation of T4 through pathways of hepatic glucuronidation and sulphation. Nonetheless it should be noted that the tT4/TBG ratio, which serves as an indirect marker of free T4 concentrations, was not elevated in the majority of those patients with high free $\mathrm{T} 4$ suggesting that the rise in fT4 may be artefactual. If however, the fT4 measurements demonstrated represent a true increase in circulating free $T 4$, our findings are in accord with the hypothesis sug 0 gested by others ${ }^{28,29}$ that the hypermetabolic state and increased oxygen consumption associated with ethanol ingestion is secondary to a 'hyperthyroid state' in the liver. The administration of the anti-thyroid drug propylthiouracil has been used clinically and reported to suppress or abolish the hypermetabolic state seen in patients with alcoholic liver disease; this treatment has also been shown to reduce circulating fT 4 and increase TSH levels. ${ }^{10}$ In primary hypothyroidism, a reduction in circulating T3 is associated with a rise in TSH. No such rise was evident in any of our patients, in agreement with previous studies of non-thyroidal illnesses. This may reflect inhibition of TSH secretion by cortisol since cortisol is a powerful inhibitor of TSH release ${ }^{30}$ This hypothesis is supported by the inverse correlation between circulating TSH and cortisol evident in the present study.

In addition to striking changes in circulating thyroid hormones, we demonstrated marked changes in levels of random cortisol in the majority of our patients with hepatitis and cirrhosis. In accord with the results of thyroid function tests, abnormal cortisol levels, determined both by direct measurement of serum cortisol and using the cortisol/CBG ratio as a measure of circulating free cortisol, were largely confined to patients with severe liver disease (hepatitis and cirrhosis). Furthermore, in accord with our observations regarding thyroid status, markedly raised cortisol concentrations were seen in the absence of clinical features of cortisol excess, 
except in one patient judged to be clinically cushingoid.

The association of 'pseudo-Cushing's syndrome' with alcohol excess is well described, ${ }^{17-19}$ although only a small number of patients has been reported, suggesting that it is relatively uncommon. The mechanism by which ethanol induces a rise in circulating cortisol ${ }^{31}$ is unclear. It has been suggested that increased plasma cortisol levels may reflect direct stimulation of the adrenal cortex by alcohol and acetaldehyde. ${ }^{32}$ In our series, however, the highest values of plasma cortisol were seen in patients with hepatitis and cirrhosis rather than in those with fatty liver, despite similar ethanol consumption in the fatty liver and hepatitis groups, suggesting that ethanol itself is not the cause of high cortisol levels, but that the presence of severe acute or chronic liver disease is more important. Correlations between circulating cortisol and tests of liver function in the present series support this view.

In contrast to the usual absence of clinical features of thyroid and adrenal steroid dysfunction despite abnormalities of circulating thyroid and adrenal hormones, hypogonadism is a well-described feature of severe liver disease, especially in males. In the present study there was little biochemical evidence of hypogonadism in females but there were striking changes in circulating testosterone levels in males, both total testosterone values and free values reflected in the testosterone/ SHBG ratio. In accord with previous studies, the majority of cirrhotic males had serum testosterone values below normal. Testosterone concentrations have been reported to fall in normal males within hours of alcohol intake ${ }^{33}$ and many features of endocrine dysfunction can be produced in experimental alcohol-fed animals in the presence of only minimally altered liver function tests. ${ }^{34}$ Furthermore, in accord with previous studies, all the cirrhotic males examined had circulating oestradiol values above normal. Circulating oestradiol levels were also high in patients with fatty liver alone, in the absence of other markers of endocrine dysfunction, which are likely therefore to reflect a direct effect of ethanol in addition to an effect of liver disease. Weakly androgenic steroids, such as androstenedione and dehydroepiandrosterone sulphate may undergo aromatization to oestrogen, a process reported to be enhanced in cirrhosis. ${ }^{35}$

In summary, it is clear from our findings that changes in these hormones are common in both those with alcoholic hepatitis and those with established cirrhosis; in general, the degree of endocrine abnormality reflects the severity of the underlying liver disease. Marked changes in circulating thyroid hormones and adrenal steroids were seen in the absence of similarly marked changes in clinical thyroid and adrenal hormone status, discrepancies which suggest that there may be an alteration in tissue responsiveness to these hormones. Both thyroid and steroid hormones act via structurally related nuclear receptor proteins ${ }^{3}$ and it is interesting to speculate that changes in expression or function of these receptors in alcoholic liver disease determine the discrepancy between circulating hormones and clinical state described above, as we have suggested previously. ${ }^{9}$ In contrast, hypogonadism was commonly associated with changes in circulating testosterone and oestradiol levels, changes being evident in some of those with minimal hepatic dysfunction, suggesting that alcohol plays a role both directly and secondarily via hepatic dysfunction in its aetiology.

\section{Acknowledgements}

We are grateful to Amerlite Diagnostics Limited for their support of these studies.

\section{References}

1. Oppenheimer, J.H., Bernstein, G. \& Surks, M.I. Increased thyroxine turnover and thyroidal function after stimulation of hepatocellular binding of thyroxine. J Clin Invest 1968, 47: 1399-1406.

2. Oppenheimer, J.H., Schwartz, H.L. \& Shapiro, H.C. Differences in primary cellular factors influencing the metabolism and distribution of 3,5,3',5-L-triiodothyronine and L-thyroxine. J Clin Invest 1970, 49: 1016-1024.

3. Oppenheimer, J.H., Schwartz, H.L., Mariash, C.N., Kinlaw, W.B., Wong, N.C.W. \& Freake, H.C. Advances in understanding of thyroid hormone action at the cellular level. Endocrine Rev 1987, 8: 288-308.

4. Sheppard, M.C. \& Ramsden, D.B. Abormalities of thyroid function tests in hospital in-patients. Postgrad Med J 1985, 61: $983-987$.

5. Franklyn, J.A., Gammage, M.D., Ramsden, D.B. \& Sheppard, M.C. Thyroid status in patients after acute myocardial infarction. Clin Sci 1984, 67: 585-590.

6. Kaplan, M.M., Larsen, P.R., Crantz, F.R., Dzau, V.J. \& Rossing, T.H. Prevalence of abnormal thyroid function test results in patients with acute medical illness. Am J Med 1982, 72: 9-16.

7. Chopra, I.J., Hershman, J.M., Pardridge, W.M. \& Nicoloff, J.T. Thyroid function in nonthyroid illness. Ann Intern Med 1983, 98: 946-957.

8. Wartofky, L. \& Burnan, K.D. Alterations in thyroid function in patients with systemic illness: the 'euthyroid sick syndrome'. End Rev 1982, 4: 164-217.

9. Williams, G.R., Franklyn, J.A., Neuberger, J.M. \& Sheppard, M.C. Thyroid hormone receptor expression in the 'sick euthyroid' syndrome. Lancet 1989, ii, 1477-1481.

10. Israel, Y., Walfish, P.G., Orrego, H., Blake, J. \& Kalant, H. Thyroid hormones in alcoholic liver disease. Effect of treatment with 6-n-Propylthiouracil. Gastroenterology 1979, 706: $116-122$. 
11. Van Thiel, D.H., Lester, R. \& Sherins, R.J. Hypogonadism in alcoholic liver disease: evidence for multiple defect. Gastroenterology 1974, 67: 1188.

12. Van Thiel, D.H. Effects of ethanol upon organ system other than the central nervous system. In: Tabakoff, B., Sutker, P.B. \& Randall, C.L. (eds) Medical and Social Aspects of Alcohol Abuse. Plenum Press, New York, 1983.

13. Lieber, C.S. Metabolic effect of ethanol and its interaction with other drugs, hepatotoxic agents, vitamins, and carcinogens: a 1988 update. Sem Liver Dis 1988, 8: 47-68.

14. Van Theil, D.H. \& Lester, R. The effect of chronic alcohol abuse on sexual function. Clin Endocrinol Metab 1979, 8: 499.

15. Van Thiel, D.H. Disorders of the hypothalamic-pituitary-gonadal axis in patients with liver disease. In: Zakim, D. \& Boyer, T.D. (eds) Hepatology, A Textbook of Liver Diseases. W.B. Saunders, Philadelphia, 1982.

16. Van Theil, D.H. \& Lester, R. Hypothalamic-pituitary-gonadal function in liver disease. Viewpoints Dig Dis 1980, 12: 13.

17. Smalls, A.G., Kloppenborg, P.W., Njo, K.T., Knoben, J.M. \& Ruland, C.M. Alcohol-induced Cushingoid syndrome. $\mathrm{Br}$ Med J 1976, ii: 1298.

18. Rees, L.H, Besser, G.M., Jeffcoate, W.J., Goldie, D.J. \& Marks, V. Alcohol-induced pseudo-Cushing's syndrome. Lancet 1977, ii: 726-728.

19. Frajria, R. \& Angeli, A. Alcohol-induced pseudo-Cushing's syndrome. Lancet 1977, i: 1050-1051.

20. Review by an International Group . Alcoholic liver disease: morphological manifestations. Lancet 1981, i: 707-711.

21. Borzio, M., Caldara, R., Borzio, F., Piepoli, V., Rampini, P. \& Ferrari, C. Thyroid function tests in chronic liver disease: evidence for multiple abnormalities despite clinical euthyroidism. Gut 1983, 24: 631-636.

22. Green, J.C., Suitcher, E.J. \& Mowat, M.A. Thyroid function and thyroid regulation in euthyroid men with chronic liver disease. Clin Endocrinol 1977, 7: 453.

23. Faber, J., Kirkengaard, C., Lumholtz, I.B., SiersbaekNielsen, K. \& Friis, T. Variations in serum T3, rT3, 3,3'-diiodothyronine and $3{ }^{\prime}, 5^{\prime}$-dioiodothyronine induced by acute myocardial infarction and propranolol. Acta Endocrinol 1980, 94: 341-345.
24. Nicoloff, J.T., Fisher, D.A. \& Appelman, J.D. The role of glucocorticoids in the regulation of thyroid function in man. J Clin Invest 1970, 49: 1922-1929.

25. Burr, W.A., Ramsden, D.B., Griffiths, R.S. \& Black, E.G. Effect of a single dose of dexamethasone on serum concentrations of thyroid hormones. Lancet 1976, ii: 58-61.

26. Westgren, U., Ahren, P., Burger, A., Ingemansson, S. \& Melander, A. Effects of dexamethasone, desoxycorticosterone and ACTH on serum concentrations of thyroxine, 3,5,3'-triiodothyronine and 3,3',5'-triiodothyronine. Acta Med Scand 1977, 202: 89-92.

27. Kahana, L., Keidar, S., Sheinfeld, M. \& Palant, A. Endogenous cortisol and thyroid hormone levels in patients with acute myocardial infarction. Clin Endocrinol 1983, 19: 131-139.

28. Hadengue, A., Moreau, R., Lee, S.S., Gaudin, C., Rueff, B. \& Lebrec, D. Liver hypermetabolism during alcohol withdrawal in humans. Role of sympathetic overactivity. Gastroenterology 1988, 94: 1047-1052.

29. Israel, Y., Videla, L. \& Bernstein, J. Hypermetabolic state after chronic ethanol consumption. Hormonal interrelations and pathogenic implications. Fed Proc 1975, 34: 2052-2059.

30. Wilber, S.F. \& Utiger, R.D. The effect of glucocorticoids on thyrotropin secretion. J Clin Invest 1969, 48: 2096-2103.

31. Wilson, N.M., Brown, P.M., Juul, S.M., Prestwich, S.A. \& Sonksen, P.H. Glucose turnover and metabolic and hormonal changes in ethanol-induced hypoglycemia. $\mathrm{Br} \mathrm{Med} J$ 1981, 282: 849-853.

32. Van Thiel, D.H. Adrenal response to ethanol: a stress response? In: Pohorecky, L.A. \& Brick, J. (eds) Stress and Alcohol Use. Elsevier, New York, 1983.

33. Van Thiel, D.H. Ethanol: its adverse effects upon the hypothalamic-pituitary-gonadal axis. J Lab Clin Med 1983 101: 21.

34. Van Thiel, D.H., Gavaler, J.S. \& Cobb, C.F. Alcohot induced testicular atrophy in the adult male rat. Endōe crinology 1979, 105: 888.

35. Olivo, J., Gordon, G.G. \& Rafii, F. Estrogen metabolism in hyperthyroidism and in cirrhosis of the liver. Steroids 1975 , 26: 47. 